\title{
Temporal metabolic response to mRNA COVID-19 vaccinations in oncology patients
}

\author{
Pooja Advani ${ }^{1} \cdot$ Saranya Chumsri ${ }^{1} \cdot$ Tanmayi Pai $^{2} \cdot$ Zhuo Li $^{3} \cdot$ Akash Sharma $^{4} \cdot$ Ephraim Parent $^{4}$ (I)
}

Received: 15 June 2021 / Accepted: 22 August 2021 / Published online: 31 August 2021

(c) The Author(s) 2021

\begin{abstract}
Background mRNA COVID-19 vaccines are known to provide an immune response seen on FDG PET studies. However, the time course of this metabolic response is unknown. We here present a temporal metabolic response to mRNA COVID-19 vaccination in oncology patients undergoing standard of care FDG PET.

Methods 262 oncology patients undergoing standard of care FDG PET were included in the analysis. 231 patients had at least one dose of mRNA COVID-19 vaccine while 31 patients had not been vaccinated. The SUVmax of the lymph nodes ipsilateral to the vaccination was compared to the contralateral to obtain an absolute change in SUVmax ( $\triangle$ SUVmax).

Results $\triangle$ SUVmax was more significant at shorter times between FDG PET imaging and COVID-19 mRNA vaccination, with a median $\Delta$ SUVmax of 2.6 (0-7 days), 0.8 (8-14 days), and 0.3 ( $>14$ days), respectively.

Conclusion Consideration should be given to performing FDG PET at least 2 weeks after the COVID-19 vaccine.
\end{abstract}

Keywords COVID-19 $\cdot$ Vaccine $\cdot$ FDG

\section{Introduction}

With rapid and widespread rollout of the mRNA COVID19 vaccinations [BNT162b2 (Pfizer-BioNTech vaccine) and mRNA-1273 (Moderna vaccine)], transient increased 2-deoxy-2 $\left[{ }^{18} \mathrm{~F}\right]$ fluoro-D-glucose (FDG) uptake in normal or enlarged ipsilateral axillary, supraclavicular, and cervical lymph nodes after vaccination has been reported in patients undergoing positron emission tomography (PET) examinations $[1,2]$. This vaccine-induced lymphadenopathy may create FDG PET/CT interpretation challenges, particularly in cancer patients, affecting assessment of cancer staging and treatment response. While vaccine-induced lymphadenopathy has been described with other vaccines

Ephraim Parent

parent.ephraim@mayo.edu

1 Department of Oncology, Mayo Clinic Florida, Jacksonville, USA

2 Department of Internal Medicine, Mayo Clinic Florida, Jacksonville, USA

3 Department of Biostatistics, Mayo Clinic Florida, Jacksonville, USA

4 Department of Radiology, Mayo Clinic Florida, 4500 San Pablo Rd S., Jacksonville, FL 32224, USA such as influenza $[3,4]$, vaccine-induced lymphadenopathy appears to be more prominent with mRNA COVID-19 vaccines. The mRNA COVID-19 vaccines are reported to be more immunogenic than traditional vaccines [5], which may influence the extent and duration of vaccine-induced lymphadenopathy on imaging. Recent literature has shed light on the confounding effects of vaccine-induced lymphadenopathy and proposed timelines for consideration of performing PET/CT examinations in relation to COVID-19 vaccine administration. However, the timeline of response and the associated differences in maximum standardized uptake value ( $\Delta$ SUVmax) between ipsilateral vaccinated axillary/subpectoral lymph nodes and contralateral lymph node chains is relatively unknown. The goal of this study is to examine the temporal impact of COVID-19 vaccine on the $\triangle$ SUVmax results obtained from FDG PET/CT and FDG PET/MRI scan in patients who received at least 1 dose of an mRNA COVID-19 vaccine compare compared to those that did not receive an mRNA COVID-19 vaccine. 


\section{Materials and methods}

A total of 262 patients, who underwent FDG PET/CT scan for work-up of cancer, cancer staging, or monitoring of therapy response at Mayo Clinic between 02/23/2021 and $04 / 16 / 2021$ were included in this prospective analysis. Information regarding COVID-19 vaccine manufacturer, date of vaccination, site of administration, and patients symptoms (current and at time of vaccination) was collected via intake questionnaires given to patients by technologists performing the scan. Patient age, sex, race, and blood sugar at time of FDG PET were obtained from the medical record as well as the type of cancer and treatments. The study has been approved by the institutional review board, (IRB \# 21-001818) and all subjects provided verbal consent and the need for written informed consent was waived. Visual and semi-quantitative analysis of metabolic response to the mRNA COVID-19 vaccine was determined by two board-certified nuclear radiologists. Patients with PET or CT evidence of lymph node metastatic involvement in the ipsilateral or contralateral axillary/sub-pectoral lymph node chains on prior FDG $\mathrm{PET} / \mathrm{CT}$ were excluded from the analysis.

Categorical variables were summarized as frequency (percentage) and continuous variables were reported as median (range). Kruskal-Walis test was used to compare continuous variables among patients with different time intervals between vaccine and PET scan while Wilcoxon rank-sum test was used to compare continuous variables between groups with and without vaccine, as well as the pairwise comparison between patients in two different time intervals between vaccine and PET scan. Chi-squared test was used to compare categorical variables between groups. Ipsilateral lymph node size and SUVmax were compared to the contralateral axillary/sub-pectoral lymph node chains to obtain an absolute difference in SUVmax $(\Delta$ SUVmax ). $\Delta$ SUVmax was calculated as the SUVmax in the vaccinated arm for vaccinated patients (or either arm for the not vaccinated patients) - SUVmax in the contralateral arm. All tests were two-sided with $p$ value $<0.05$ considered statistically significant. To account for multiple comparison, Bonferroni correction was used for the pairwise comparison between different time intervals. Linear regression models were used to identify univariable and multivariable predictors for the between-arms difference in SUV max difference. The analysis was done using R3.6.2 [6].

\section{Results}

285 oncology patients undergoing standard of care FDG PET/CT or FDG PET/MRI, and who had prior FDG PET studies for comparison, were initially included in this study. 23 of these patients were excluded due to PET or CT evidence of known metastatic disease in the axillary/sub-pectoral lymph node chains on the prior FDG PET/CT examination. Median age of the remaining 262 patients was 70 (range 20-94) years and 231 patients had at least one dose of COVID-19 vaccine before the PET scan (median age: 71 years) while 31 patients did not receive the vaccine (median age: 59 years). Median blood sugar of the 31 unvaccinated patients was $104 \mathrm{mg} /$ $\mathrm{dL}$ (range 77-214 $\mathrm{mg} / \mathrm{dL}$ ) and the median blood sugar of the 231 patients that were vaccinated was $103 \mathrm{mg} / \mathrm{dL}$ (range $68-213 \mathrm{mg} / \mathrm{dL}$ ). Of the vaccinated and non-vaccinated patients, $51 \%$ and $48 \%$ were male, respectively, and $76 \%$ of non-vaccinated and $91 \%$ of vaccinated patients were Caucasian.

Of the 231 vaccinated patients, 113 (47\%) were noted to have visually increased FDG uptake in the ipsilateral axillary/sub-pectoral lymph nodes in the PET scan compared to the contralateral. When stratified by time since vaccination, $70 \%(23 / 33)$ of patients who received the vaccine between 0 and 7 days prior to PET had visually evident uptake, which dropped to $55 \%(21 / 38)$ at $8-14$ days and $44 \%(71 / 160)$ when greater than 2 weeks since vaccination. There was no substantial difference between patients undergoing FDG PET/CT $>2$ weeks after vaccination ( $>14$ days) compared to those that waited more than 4 weeks (>28 days) with $40 \%$ (31/78) of patients in this last group demonstrating visually appreciated ipsilateral FDG uptake.

Semi-quantitative analysis confirmed visual findings with a median $\triangle$ SUVmax of 0.1 for patients without vaccine versus 0.4 for all patients with vaccine $(p<0.001$, Table 1$)$ regardless of time since vaccination. The greatest $\Delta$ SUVmax for an unvaccinated patient was 2.7. Time between COVID19 vaccine and PET scan was associated with statistically significant changes in $\triangle$ SUVmax but remained greater than non-vaccinated patients at all time points. $\Delta$ SUVmax was greater at shorter time intervals between the dates of the COVID-19 vaccination with a median $\Delta$ SUVmax of 2.6 (0-7 days), 0.8 (8-14 days), and 0.3 (> 14 days), respectively (Fig. 1 and Table 2). Of the 160 patients that received a mRNA COVID-19 vaccination greater than 2 weeks prior to FDG PET, 12 patients demonstrated a $\triangle$ SUVmax $>5.0$ (7.5\%; Fig. 2). Additional quantitative analysis found non-significant differences in $\triangle$ SUVmax in vaccinated patients when comparing those patients that received vaccine $>14$ days from FDG PET examination as compared 
Table 1 Between-arms difference in SUV max ( $\Delta$ SUVmax) based on receipt of vaccine

\begin{tabular}{|c|c|c|c|c|}
\hline & $N(N=31)$ & $Y(N=231)$ & Total $(N=262)$ & $p$ value \\
\hline Age & & & & $<0.001$ \\
\hline$N$ & 31 & 231 & 262 & \\
\hline Median (range) & $59.00(20.00,92.00)$ & $71.00(20.00,94.00)$ & $70.00(20.00,94.00)$ & \\
\hline Gender & & & & 0.78 \\
\hline $\mathrm{F}$ & $16(51.6 \%)$ & $113(48.9 \%)$ & $129(49.2 \%)$ & \\
\hline M & $15(48.4 \%)$ & $118(51.1 \%)$ & $133(50.8 \%)$ & \\
\hline Race & & & & 0.013 \\
\hline Non-Caucasian & $7(24.1 \%)$ & $20(8.9 \%)$ & $27(10.7 \%)$ & \\
\hline Caucasian & $22(75.9 \%)$ & $204(91.1 \%)$ & $226(89.3 \%)$ & \\
\hline $\begin{array}{l}\text { Between-arms dif- } \\
\text { ference in SUV } \\
\max \end{array}$ & & & & $<0.001$ \\
\hline$N$ & 31 & 231 & 262 & \\
\hline Median (range) & $0.10(-0.80,2.70)$ & $0.40(-2.00,19.30)$ & $0.30(-2.00,19.30)$ & \\
\hline Blood sugar & & & & 1.00 \\
\hline$N$ & 31 & 225 & 256 & \\
\hline Median (range) & $104.00(77.00,14.00)$ & $103.00(68.00,213.00)$ & $103.50(68.00,14.00)$ & \\
\hline SUV/blood sugar & & & & 0.065 \\
\hline$N$ & 30 & 225 & 255 & \\
\hline Median (range) & $0.01(0.00,0.05)$ & $0.01(0.00,0.29)$ & $0.01(0.00,0.29)$ & \\
\hline
\end{tabular}

Fig. 1 Box plot diagram of FDG PET $\Delta$ SUVmax values [vaccinated arm for vaccinated patients (or either arm for the not vaccinated patients) - SUVmax in the contralateral arm] in vaccinated and non-vaccinated patients] and days between vaccinations

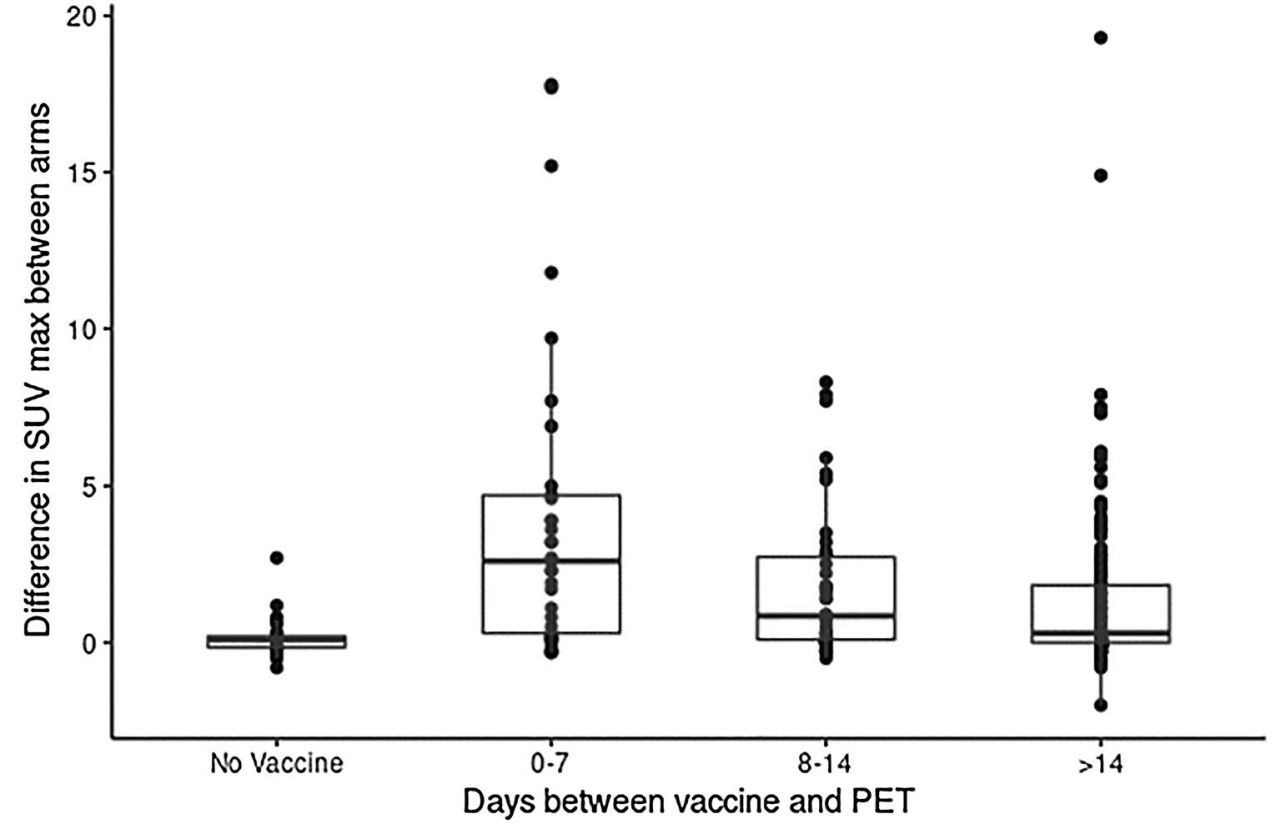

to $>28$ days (Supplemental). After adjusting for the presence of symptoms, $\Delta$ SUVmax retained greater significance at shorter intervals and the presence or absence symptoms were not found to be an independent variable for $\triangle \mathrm{SUV}$ max. Interestingly, of the 262 patients analyzed, only 5 were found to have enlarged axillary/sub-pectoral lymph nodes with a short axis $>1.0 \mathrm{~cm}$ and 4 of 5 reported symptoms including sore arm and flu-like symptoms. Also, 4 of the 5 received the mRNA vaccine within 7 days (range 3-7 days) of the FDG PET examination and had an average $\Delta$ SUVmax of 8.1 (range 3.2-17.7). The remaining patient with an enlarged axillary lymph node received the COVID-19 vaccination 30 days prior and had a $\Delta$ SUVmax of 1.7. Sub-analysis of blood sugar, age, race and gender found minor associations with $\triangle$ SUVmax; however, time from vaccine retained the most significant variable of FDG uptake (Supplemental). 
Table 2 Patient characteristics and $\Delta$ SUVmax according to days from vaccinations

\begin{tabular}{|c|c|c|c|c|}
\hline & $0-7(N=33)$ & $8-14(N=38)$ & $>14(N=160)$ & $p$ value \\
\hline Days from vaccine & & & & $<0.001$ \\
\hline$N$ & 33 & 38 & 160 & \\
\hline Median (range) & $5.0(0.0,7.0)$ & $11.5(8.0,14.0)$ & $28.0(15.0,93.0)$ & \\
\hline$\Delta$ SUVmax & & & & $<0.001$ \\
\hline$N$ & 33 & 38 & 160 & \\
\hline Median (range) & $2.6(-0.3,17.8)$ & $0.8(-0.5,8.3)$ & $0.3(-2.0,19.3)$ & \\
\hline Dose & & & & $<0.001$ \\
\hline 1 & $14(42.4 \%)$ & $17(44.7 \%)$ & $26(16.2 \%)$ & \\
\hline 2 & $19(57.6 \%)$ & $21(55.3 \%)$ & $134(83.8 \%)$ & \\
\hline Maker & & & & 0.023 \\
\hline MODERNA & $20(60.6 \%)$ & $13(35.1 \%)$ & $94(59.5 \%)$ & \\
\hline PFIZER & $13(39.4 \%)$ & $24(64.9 \%)$ & $64(40.5 \%)$ & \\
\hline Symptoms & & & & 0.068 \\
\hline No & $28(84.8 \%)$ & $35(92.1 \%)$ & $153(95.6 \%)$ & \\
\hline Yes & $5(15.2 \%)$ & $3(7.9 \%)$ & $7(4.4 \%)$ & \\
\hline
\end{tabular}

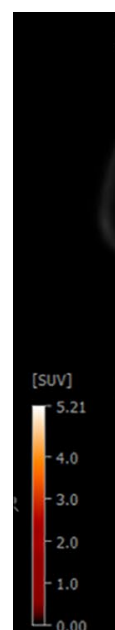

\section{A}
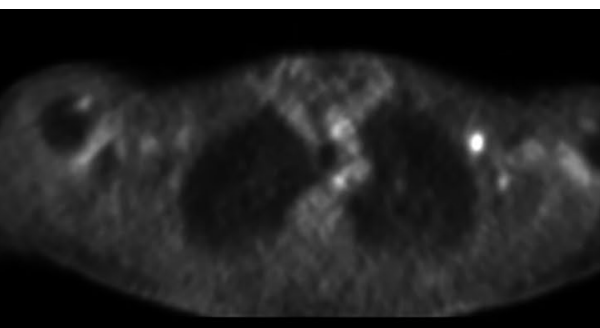

D

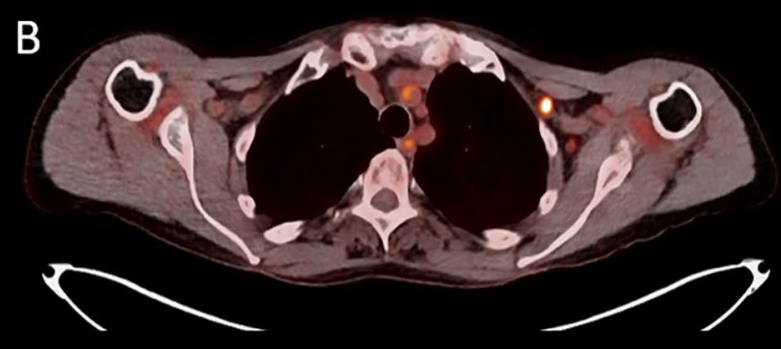

C

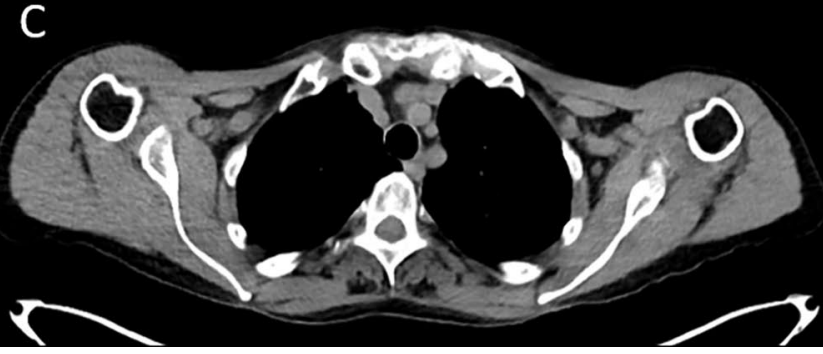

Fig. 2 Selected images from serial FDG PET/CT examinations for a patient who received 2 doses of Moderna vaccination, with the second dose approximately 1 month before initial imaging. Trans-axial FDG PET (A) fused FDG PET/CT (B) transaxial non-contrast CT image (C) and FDG PET MIP (D) show two non-enlarged but hypermetabolic left axillary lymph nodes, ipsilateral to the COVID-19 vaccination sites in the left arm. $\Delta$ SUVmax was 6.7 and 6.8 , in the anterior and posterior nodes, respectively 


\section{Discussion}

Based on previous experience with routine vaccinations, vaccine-related nodal FDG uptake typically occurs within 7 days of vaccination and generally subsides by 12-14 days [7]. Studies have suggested performing PET/CT at least 2 weeks after vaccination in cancer patients for which interpretation is anticipated to be potentially impacted by the vaccination, though some have suggested to wait 4-6 weeks after vaccination [1]. Our data demonstrate a progressive decrease in SUV max after the first week from vaccine, and the lowest $\Delta$ SUVmax being seen after 2 weeks from vaccination, with non-substantial changes between patients waiting either $>2$ weeks or $>4$ weeks after vaccination. However, it should be noted that even after 2 weeks, there is a statistically significant $\Delta$ SUVmax for vaccinated patients compared to non-vaccinated patients, with a few outlying patients demonstrating markedly increased FDG uptake (Fig. 1). This is in line with the reported late-term metabolic response (7-10 weeks) after mRNA vaccination [8]. It has been postulated that symptomatic patients would have a greater radiographic response. However, our results demonstrate that self-reported symptoms do not affect the likelihood of having metabolically active nodal findings. Additionally, it should be noted that the vast majority of patients that received the vaccine did not have lymphadenopathy $>1.0 \mathrm{~cm}$ on the short axis despite many having increased FDG uptake (Fig. 3). This discrepancy between metabolic response (FDG PET) and lymphadenopathy has been previously reported and can be considered a hallmark of mRNA vaccination response and may help guide the interpreting physician to distinguish between a pronounced COVID-19 vaccination response and true nodal metastatic disease [9].

\section{Conclusion}

Due to the relative recent and novel mRNA COVID-19 vaccinations, radiologists and oncologists are facing a diagnostic challenge in deciphering axillary, cervical and sub-pectoral lymphadenopathy in patients with active cancer or those that are undergoing work-up for cancer. Based on our study, consideration should be given to performing FDG PET/CT at least 2 weeks after the COVID-19 vaccine, if it does not interfere with the care of the patient. However, our findings also demonstrate that a small number of patients will

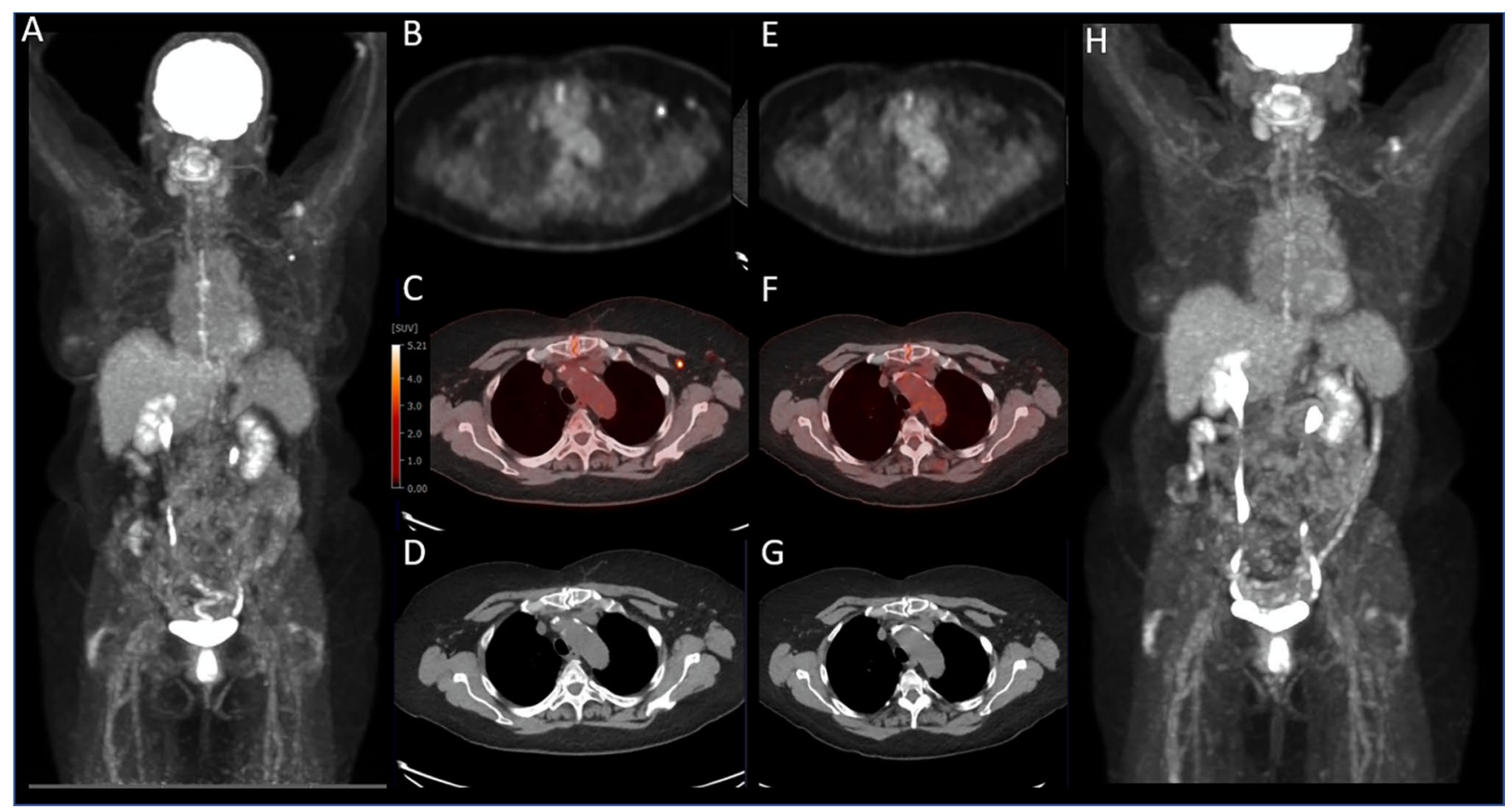

Fig. 3 Selected images from serial FDG PET/CT examination for a patient who received 1 dose of Pfizer vaccination 11 days before initial imaging, as well as a second FDG PET/CT approximately 3 months later for clinical oncologic follow-up, with interval administration of the second dose of COVID-19 vaccination. FDG PET MIP (A) trans-axial FDG PET (B) fused FDG PET/CT (C) and trans-axial non-contrast CT images show a non-enlarged but hypermetabolic left axillary lymph node; $\Delta \mathrm{SUVmax}=5.9$. On the subsequent evaluation, FDG PET MIP (H) trans-axial FDG PET (E) fused FDG PET/ CT $(\mathbf{F})$ and trans-axial non-contrast CT images FDG PET $(\mathbf{G})$ images at matching levels show resolution of FDG uptake in the same-sized lymph node, now seen only on the CT image 
continue to have a durable metabolic response which persists even after 2 weeks. Further studies regarding the optimal timing, and recommendations for follow-up of abnormal FDG PET/CT scan after COVID vaccine are needed.

Supplementary Information The online version contains supplementary material available at https://doi.org/10.1007/s12149-021-01675-8.

\section{Declarations}

Conflict of interest No funding was obtained for this study. Dr. Pooja Advani received research funding from Genentech. Dr. Saranya Chumsri receives research funding from Merck and Pfizer. Dr. Ephraim Parent receives research funding from Blue Earth Diagnostics. The other authors declare that they have no competing interests.

Ethics approval and consent to participate All procedures performed in studies involving human participants were in accordance with the ethical standards of the institutional and/or national research committee and with the 1964 Helsinki declaration and its later amendments or comparable ethical standards. The recruitment protocol was approved by the Institutional Review Board (IRB) and complied with the Health Insurance Portability and Accountability Act (HIPPA). Informed consent was obtained from all individual participants included in the study.

Open Access This article is licensed under a Creative Commons Attribution 4.0 International License, which permits use, sharing, adaptation, distribution and reproduction in any medium or format, as long as you give appropriate credit to the original author(s) and the source, provide a link to the Creative Commons licence, and indicate if changes were made. The images or other third party material in this article are included in the article's Creative Commons licence, unless indicated otherwise in a credit line to the material. If material is not included in the article's Creative Commons licence and your intended use is not permitted by statutory regulation or exceeds the permitted use, you will need to obtain permission directly from the copyright holder. To view a copy of this licence, visit http://creativecommons.org/licenses/by/4.0/.

\section{References}

1. McIntosh LJ, Bankier AA, Vijayaraghavan GR, Licho R, Rosen MP. COVID-19 vaccination-related uptake on FDG PET/CT: an emerging dilemma and suggestions for management. AJR Am J Roentgenol. 2021. https://doi.org/10.2214/AJR.21.25728.

2. Doss M, Nakhoda SK, Li Y, Yu JQ. COVID-19 vaccine-related local FDG uptake. Clin Nucl Med. 2021;46(5):439-41.

3. Shirone N, Shinkai T, Yamane T, Uto F, Yoshimura H, Tamai H, et al. Axillary lymph node accumulation on FDG-PET/CT after influenza vaccination. Ann Nucl Med. 2012;26(3):248-52.

4. Thomassen A, Lerberg Nielsen A, Gerke O, Johansen A, Petersen H. Duration of 18 F-FDG avidity in lymph nodes after pandemic H1N1v and seasonal influenza vaccination. Eur J Nucl Med Mol Imaging. 2011;38(5):894-8.

5. Prendecki M, Clarke C, Edwards H, McIntyre S, Mortimer P, Gleeson S, et al. Humoral and T-cell responses to SARS-CoV-2 vaccination in patients receiving immunosuppression. Ann Rheum Dis. 2021. https://doi.org/10.1136/annrheumdis-2021-220626.

6. Team RDC. R: a language and environment for statistical computing. R Foundation for Statistical Computing, Vienna, Austria: R Foundation for Statistical Computing. 2019. https://www.R-proje ct.org/. Accessed 2 Aug 2021.

7. Panagiotidis E, Exarhos D, Housianakou I, Bournazos A, Datseris I. FDG uptake in axillary lymph nodes after vaccination against pandemic (H1N1). Eur Radiol. 2010;20(5):1251-3.

8. Eshet Y, Tau N, Alhoubani Y, Kanana N, Domachevsky L, Eifer M. Prevalence of increased FDG PET/CT axillary lymph node uptake beyond 6 weeks after mRNA COVID-19 vaccination. Radiology. 2021. https://doi.org/10.1148/radiol.2021210886: 210886.

9. Schroeder DG, Jang S, Johnson DR, Takahashi H, Navin PJ, Broski SM, et al. Frequency and characteristics of nodal and deltoid FDG and 11C-choline uptake on PET imaging performed after COVID-19 vaccination. AJR Am J Roentgenol. 2021. https://doi. org/10.2214/AJR.21.25928.

Publisher's Note Springer Nature remains neutral with regard to jurisdictional claims in published maps and institutional affiliations. 